\title{
Video Article \\ Optimized System for Cerebral Perfusion Monitoring in the Rat Stroke Model of Intraluminal Middle Cerebral Artery Occlusion
}

\author{
Simone Beretta ${ }^{1}$, Matteo Riva ${ }^{1}$, Davide Carone ${ }^{1}$, Elisa Cuccione ${ }^{1}$, Giada Padovano ${ }^{1}$, Virginia Rodriguez Menendez ${ }^{1}$, Giovanni B. Pappadá ${ }^{1}$, \\ Alessandro Versace ${ }^{1}$, Carlo Giussani ${ }^{1}$, Erik P. Sganzerla ${ }^{1}$, Carlo Ferrarese ${ }^{1}$ \\ ${ }^{1}$ Department of Neuroscience and Biomedical Technologies, University of Milano Bicocca
}

Correspondence to: Simone Beretta at simone.beretta@unimib.it

URL: https://www.jove.com/video/50214

DOI: doi: $10.3791 / 50214$

Keywords: Medicine, Issue 72, Neuroscience, Neurobiology, Biomedical Engineering, Anatomy, Physiology, Surgery, Brain Ischemia, Stroke, Hemodynamics, middle cerebral artery occlusion, cerebral hemodynamics, perfusion monitoring, Laser Doppler, intracranial collaterals, ischemic penumbra, rat, animal model

Date Published: 2/17/2013

Citation: Beretta, S., Riva, M., Carone, D., Cuccione, E., Padovano, G., Rodriguez Menendez, V., Pappadá, G.B., Versace, A., Giussani, C., Sganzerla, E.P., Ferrarese, C. Optimized System for Cerebral Perfusion Monitoring in the Rat Stroke Model of Intraluminal Middle Cerebral Artery Occlusion. J. Vis. Exp. (72), e50214, doi:10.3791/50214 (2013).

\section{Abstract}

The translational potential of pre-clinical stroke research depends on the accuracy of experimental modeling. Cerebral perfusion monitoring in animal models of acute ischemic stroke allows to confirm successful arterial occlusion and exclude subarachnoid hemorrhage. Cerebral perfusion monitoring can also be used to study intracranial collateral circulation, which is emerging as a powerful determinant of stroke outcome and a possible therapeutic target. Despite a recognized role of Laser Doppler perfusion monitoring as part of the current guidelines for experimental cerebral ischemia, a number of technical difficulties exist that limit its widespread use. One of the major issues is obtaining a secure and prolonged attachment of a deep-penetration Laser Doppler probe to the animal skull. In this video, we show our optimized system for cerebral perfusion monitoring during transient middle cerebral artery occlusion by intraluminal filament in the rat. We developed in-house a simple method to obtain a custom made holder for twin-fibre (deep-penetration) Laser Doppler probes, which allow multi-site monitoring if needed. A continuous and prolonged monitoring of cerebral perfusion could easily be obtained over the intact skull.

\section{Video Link}

The video component of this article can be found at https://www.jove.com/video/50214/

\section{Introduction}

Translational research on hemodynamic factors affecting stroke pathophysiology and therapy needs to be implemented, since this important issue is often paradoxically neglected by basic research studies ${ }^{1}$.

Cerebral perfusion monitoring is an essential, but underused, tool for accurate ischemic stroke modeling ${ }^{2}$. Aside from the confirmation of arterial vessel occlusion and exclusion of subarachnoid hemorrhage ${ }^{3}$, continuous cerebral perfusion monitoring may provide useful data about the degree and consistency of perfusion deficit, the functional status of intracranial collateral vessels and the hemodynamic effect of new therapeutic approaches.

A recent study from our group indicate that multi-site hemodynamic monitoring may be used to assess intracranial collateral circulation and can predict infarct size and functional deficit ${ }^{4}$. These experimental findings are consistent with clinical studies which showed that the functional performance of cerebral collateral circulation is predictive of clinical outcome in ischemic stroke patients ${ }^{5,6}$. For this reason, cerebral collaterals have been advocated as a potential therapeutics target in the acute phase of ischemic stroke ${ }^{7}$.

Laser-Doppler (LD) instruments are the most common tool used to measure cerebral perfusion in experimental ischemic stroke and their use is recommended by recent guidelines on this theme ${ }^{8}$. LD instruments measure microvascular perfusion in a small cortical volume, the depth of the recorded signal being dependent on the width of fibre separation, with twin fibre LD probes allowing a deeper penetration compared to single fibre LD probes ${ }^{9}$. Blood flow values are expressed as arbitrary perfusion units (P.U.) which indicate relative rather than absolute cerebral blood flow. Calibration of P.U. is usually performed using motility standards, according to manufacturer's instructions. LD flowmetry allows a continuous dynamic monitoring and generation of quantitative data within the same session.

Among the technical problems that currently limit the use of LD, a major issue is obtaining a secure and prolonged attachment of a deeppenetration Laser Doppler probe to the animal skull. This is essential for prolonged monitoring and if multiple probes are used for different cerebral arterial territories, as we are performing in our laboratory.

In particular, prolonged surgical time is required if probes are attached to the skull using burr holes or cranial screws, whereas poor signal and unsecure attachment occurs if single fibre (low-penetration) LD probes are attached to the skull by simple surgical glue. Twin fibre (deep- 
penetration) LD probes provide a higher and more consistent signal, but they are larger than single fibre probes and cannot be attached to the skull using surgical glue only.

In this video, we show our optimized system for cerebral perfusion monitoring during transient middle cerebral artery occlusion by intraluminal filament in the rat. We describe a simple method to obtain an efficient, custom made, low cost holder for single or multiple twin-fibre (deeppenetration) LD probes, to be used for prolonged monitoring of cerebral perfusion over the intact skull.

The surgical procedure for transient MCAO in the rat could be seen in the video-article by Uluç and coworkers ${ }^{10}$ and is not shown in this video.

\section{How to Make the Probe Holder (Single Site or Multi-site)}

1. The materials required are natural rubber, small plastic tubes and a metal stylet. The probe holder can be customized to the size of the animal, the number and size of the Laser Doppler probes, and the cerebral vascular territory which needs to be monitored.

2. Cut the natural rubber of the needed size (approximately $10 \mathrm{~mm} \times 10 \mathrm{~mm}$ for a $300 \mathrm{~g}$ rat).

3. Mark the positions of the probe(s) and the bregma on the natural rubber, according to the desired stereotaxic coordinates; for middle cerebral artery occlusion, the typical coordinates for the ischemic core are expected at bregma $-1 \mathrm{~mm}, 5 \mathrm{~mm}$ lateral to the midline; for a peripheral ischemic borderzone territory, the expected coordinates can be at bregma $+2 \mathrm{~mm}, 2 \mathrm{~mm}$ lateral to the midline.

4. Mark very clearly the position of the bregma with an X, this is the landmark for attaching the probe holder to the skull.

5. Insert the stylet into a small plastic tube (its size needs to match the size of the probe).

6. Insert the stylet into the natural rubber at the point where the probe has to be positioned; push the stylet into the rubber until the plastic tube has been inserted into the rubber too.

7. Withdraw the stylet.

8. If multiple probes are needed, repeat steps 1.5-1.7 for other probe positions.

9. Optional for multiple probes: wrap a tape around the plastic tubes to ensure a better stabilization of the probes.

10. Optional: perform chemical sterilization of several probe holders for future uses.

\section{Presurgical Preparation}

1. MCAO stroke model is usually performed as a survival surgery. In this case, as shown in our video (survival time $24 \mathrm{hr}$ post-surgery), the surgeon uses an aseptic technique with sterilized instruments and supplies.

2. Anesthetize the rat with isoflurane ( $3 \%$ induction phase, $1.5 \%$ maintenance).

3. Place the rat in prone position on the operating table.

4. Gently shave the head of the rat.

5. Apply an antiseptic solution to a gauze pad and disinfect the skin.

6. Administer Lidocaine $2 \% 5 \mathrm{mg} / \mathrm{kg}$ subcutaneously in the cranial area.

\section{Probe Positioning and Securing Over the Intact Skull}

1. Make a right paramedian skin incision (for right $\mathrm{MCAO}$ ) and dissect the subcutaneous tissue to reach the cranial fascia (galea aponeurotica).

2. Make a right paramedian incision in the cranial fascia and perform blunt dissection to reach the skull bone; prepare a skull bone area which is large enough for the application of the probe holder.

3. Please note: no need for drilling or bone thinning.

4. Apply Merbromin solution to disinfect and dry the skull surface.

5. Use a hair dryer (set for cold air) to accelerate drying the skull surface.

6. At this point, the cranial sutures and the bregma are clearly visible.

7. Customize the probe holder by cutting the edges with sterile scissors.

8. Apply a small quantity of surgical glue (Cyanoacrylate, veterinary-approved) to the surface underneath the probe holder, carefully avoiding the inferior opening of the plastic tubes (please note: if a significant amount of glue remains between the optical surface of the probe and the skull, this may produce a low-signal and may damage the probe after multiple uses).

9. Apply the probe holder to the skull surface, carefully matching the bregma with the X landmark. Apply gentle pressure over the probe holder.

10. Use a hair dryer (set for cold air) to accelerate drying the surgical glue.

11. Secure the probe holder by tying a surgical thread around the probe holder and the head of the animal; be careful to position the surgical thread over the mandibula, avoiding the submandibular region and the neck.

12. Optional: fill the plastic tube(s) of the probe holder with an optical gel (e.g. a common ultrasound or electrocardiography gel); this will increase the quality of the LD signal.

13. Place the probe(s) in the probe holder and verify the actual reading of the LD flowmeter. Use the LD flowmeter according to manufacturer's instructions.

14. Secure the probe(s) tying them around head of the animal; be careful to position the surgical thread over the mandibula, avoiding the submandibular region and the neck.

\section{Cerebral Perfusion Monitoring During MCAO}

1. Place the rat in the supine position, carefully avoiding tractional forces on the probe(s) or probe holder.

2. Start cerebral perfusion monitoring during MCAO. 


\section{Removal of the Probe(s) and Probe Holder}

1. Cut the sutures around the probe(s), the probe holder and the head of the animal.

2. Gently blunt dissect cranial soft tissues and skin around the natural rubber base of the probe holder.

3. Remove the probe holder.

4. Apply an antiseptic solution to the skull surface.

5. Suture the cranial skin.

\section{Post-operative Care}

1. Administer $2.5 \mathrm{ml}$ of saline solution subcutaneously to prevent dehydration and keep the animal warm using a heating pad after stopping gaseous anesthesia.

2. For survival surgery: provide preemptive analgesia with Ketoprofen $4 \mathrm{mg} / \mathrm{kg}$ subcutaneously and repeat the same dose at $12 \mathrm{hr}$ postoperatively

3. Under our experimental conditions, euthanasia was performed at $24 \mathrm{hr}$ post-surgery by $\mathrm{CO}_{2}$ inhalation.

\section{Representative Results}

Transient MCAO (60 min) was induced by inserting a silicone-coated filament in the external carotid artery. The filament was then pushed through the terminal end of the internal carotid artery up to the origin of the MCA, under LD monitoring. Common carotid artery and pterygopalatin artery were transiently occluded during the surgical insertion of the filament. A schematic representation of the surgical procedure is shown in Figure 1A.

The cranial coordinates for positioning the two LD probes were chosen according to the underlying arterial territory. Preliminary experiments with gelatin-ink perfusion (Figure 1B) showed that the ischemic core is expected in the central MCA territory (bregma -1 mm, $5 \mathrm{~mm}$ from midline; Probe 1), while the collateral flow is expected in the borderzone territory between the cortical branches of middle and anterior cerebral arteries (bregma +2 mm, 2 mm from midline; Probe 2)

Cerebral hemodynamics was studied using multi-site Laser Doppler probes during the entire period of the surgical procedure, i.e. before, during and after MCAO (Figure 2). The cerebral perfusion deficit during MCAO was smaller and showed a higher degree of variability in Probe 2 compared to Probe 1, suggesting inter-individual differences in the functional performance of intracranial collaterals under ischemic conditions. The multi-site Laser Doppler monitoring also allows to study the cerebral hemodynamic changes during the occlusion of proximal extra-cranial cerebral arteries (common carotid artery, internal carotid artery, pterygopalatin artery).

Stroke outcome was assessed $24 \mathrm{hr}$ after reperfusion by infarct volume, calculated on 19 consecutive sections stained with Cresyl violet (Figure 3 ), and Garcia functional neuroscore ${ }^{11}$. Immunohistochemistry for specific markers associated with ischemic brain injury was performed, in order to obtain a topographical distribution of neuronal loss (microtubule associated protein 2, MAP2) and ischemic penumbra (heat shock protein-70, Hsp70) in relation to the multi-site hemodynamic monitoring of intracranial circulation (Figure 4). 

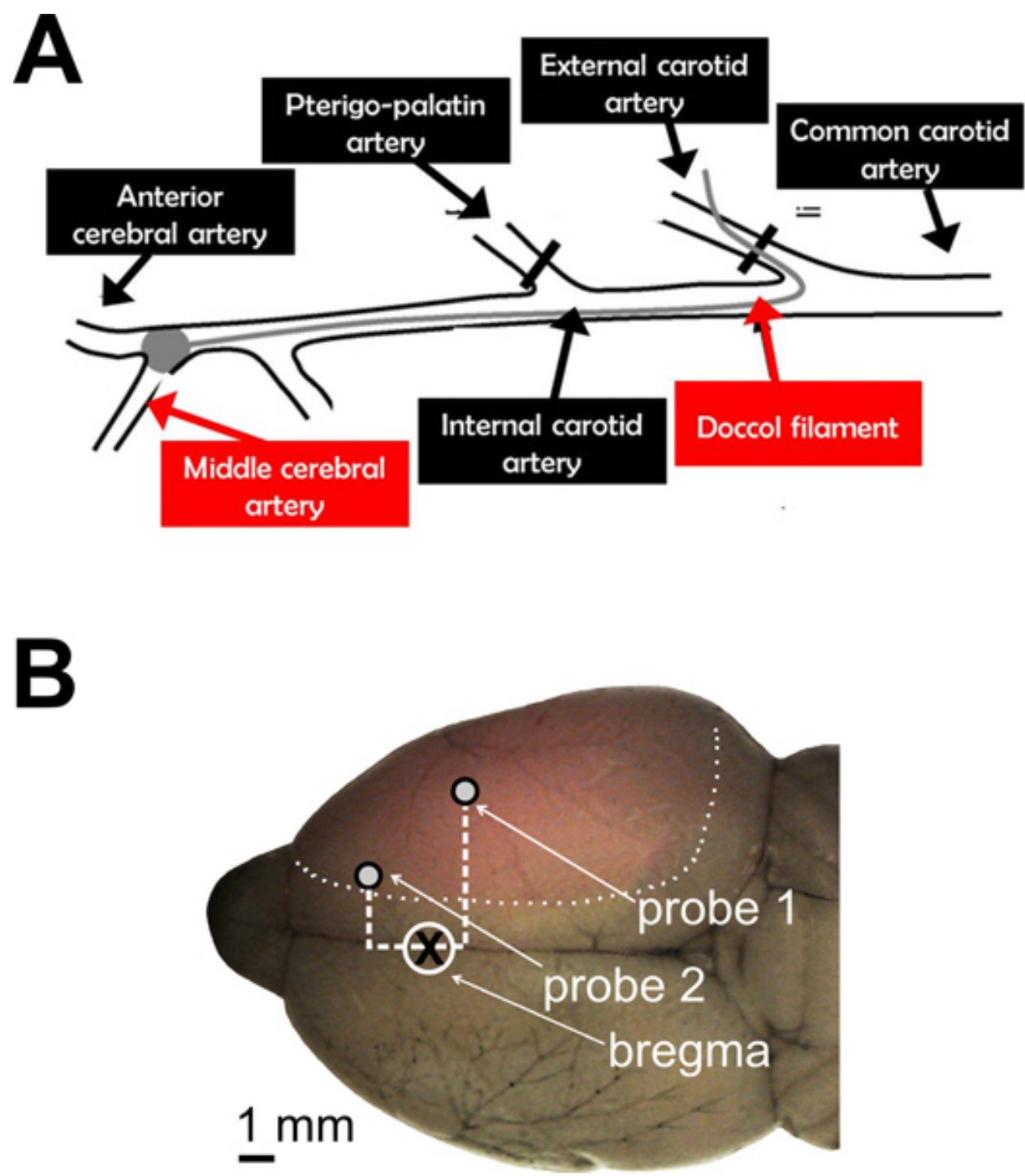

Figure 1. Cerebral perfusion monitoring during intraluminal MCAO in the rat. A. Schematic representation of the surgical procedure for transient MCAO. A silicone-coated filament was used to occlude the origin of the MCA, after being introduced in the external carotid artery and pushed through the internal carotid artery. Proximal cervical arteries were either ligated (external carotid artery) or transiently occluded (pterygopalatin artery and common carotid artery) during the procedure. B. A representative brain is shown after gelatin-ink staining. Transcardiac perfusion of gelatin-ink solution were performed $60 \mathrm{~min}$ after the onset of ischemia, without reperfusion. The normally perfused brain was stained by gelatin-ink and appeared as grey-colored with black stained vessels, while the ischemic (not-perfused) area remained unstained (pink-colored). Cranial coordinates for positioning the two LD probes are shown. Probe $1=-1 \mathrm{~mm}$ from bregma, $5 \mathrm{~mm}$ from midline; Probe 2 = +2 $\mathrm{mm}$ from bregma, $2 \mathrm{~mm}$ from midline. 


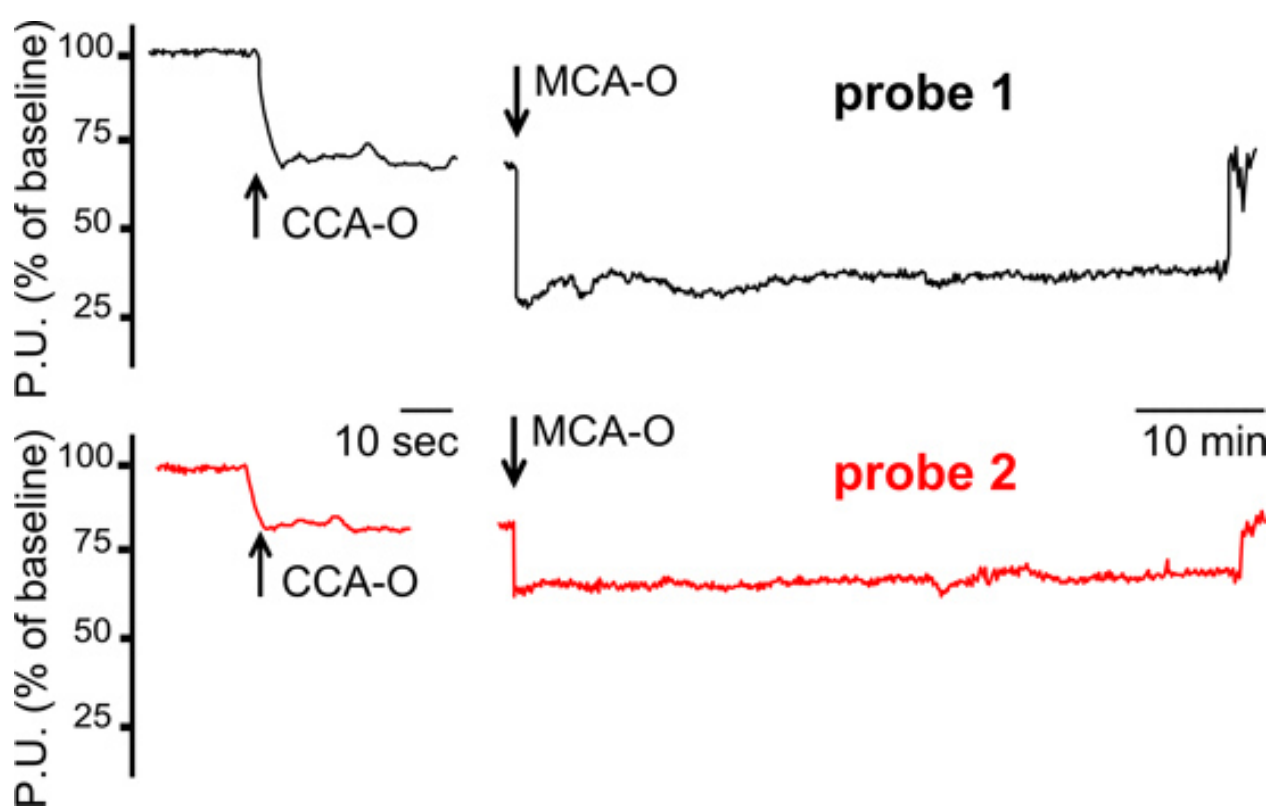

Figure 2. Cerebral hemodynamic recordings using multi-site Laser Doppler probes. A typical hemodynamic pattern which suggests functionally active intracranial collaterals under ischemic conditions is shown. In this animal, LD tracings showed a smaller perfusion deficit in Probe 2 channel, compared to Probe 1 channel, during both CCA occlusion and MCA occlusion. MCA-O=middle cerebral artery occlusion. CCA$\mathrm{O}=$ common carotid artery occlusion. P.U.= perfusion units.

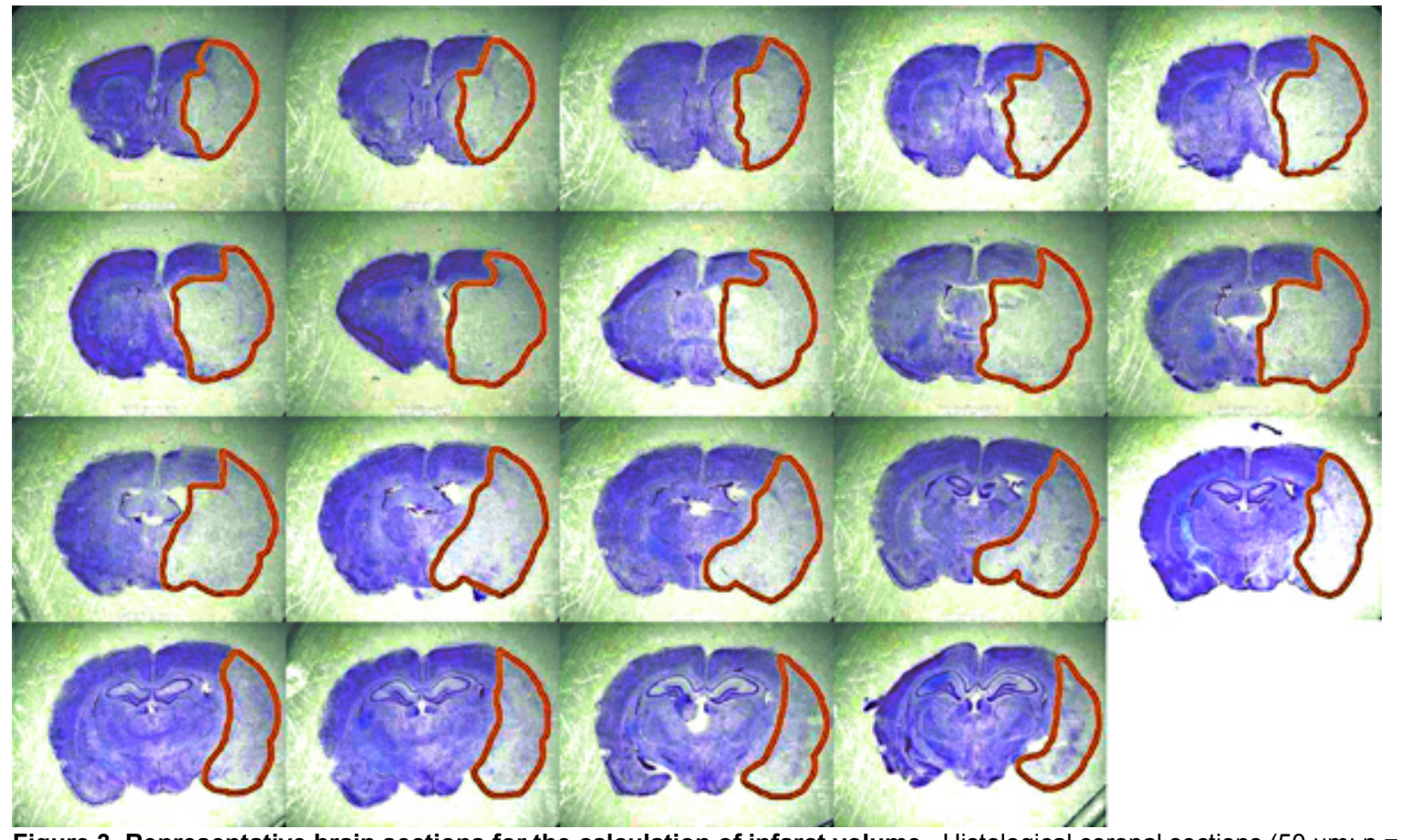

Figure 3. Representative brain sections for the calculation of infarct volume. Histological coronal sections $(50 \mu \mathrm{m} ; \mathrm{n}=19 \mathrm{with} 250 \mu \mathrm{m}$ interval; bregma $+2.5 \mathrm{~mm}$ to $-3.0 \mathrm{~mm}$ ) are fixed in $4 \%$ paraformaldehyde and stained with Cresyl violet $0.1 \%$. Infarct volume is calculated using Image J image processing software, corrected for inter-hemispheric asymmetries due to cerebral edema, and expressed in $\mathrm{mm}^{3}$. Click here to view larger figure 

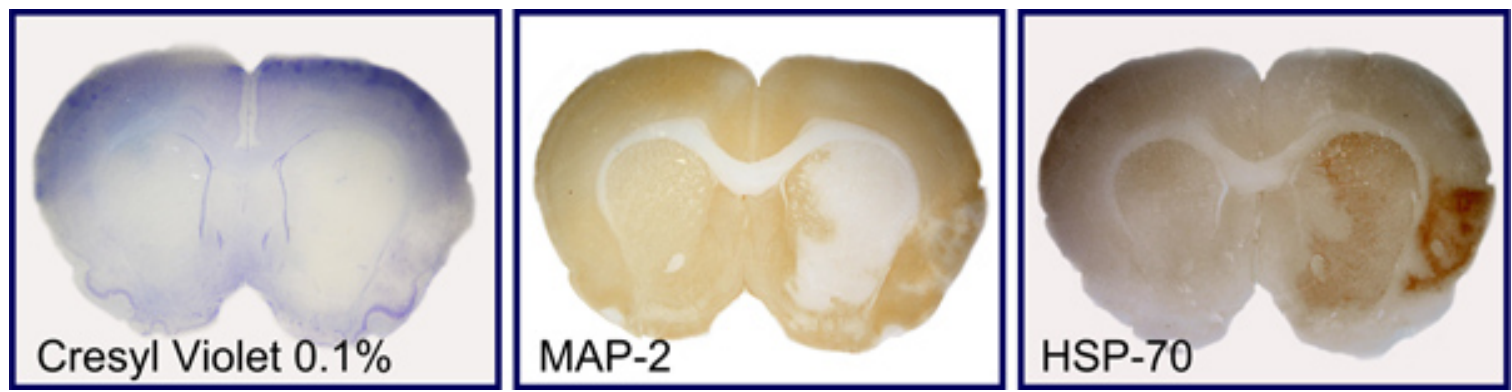

Figure 4. Immunostaining of molecular markers of neuronal loss and penumbra. Representative consecutive brain sections are shown, which were stained with Cresyl violet $0.1 \%(\mathbf{A})$ or immunostained with markers of neuronal loss (microtubule associated protein 2, MAP2; B) and ischemic penumbra (heat shock protein-70, Hsp70; C).

\section{Discussion}

We developed in-house a simple and low-cost system for a secure attachment of one or multiple twin-fibre (deep penetration) LD probes to the intact skull of rats during the MCAO procedure. Albeit apparently a trivial problem, obtaining a reliable attachment of the LD probe to the skull is actually a major issue in this experimental setting, since it is the prerequisite for a smooth signal detection and a successful monitoring of cerebral perfusion.

Invasive procedures, such as burr holes and bone screws, usually prolong the surgical time and introduce more experimental variables related to craniotomy, and this may discourage researchers and refrain them from using LD monitoring. On the other hand, the use of single fibre (low penetration) probes, which are thinner and relatively easier to be glued directly to the skull surface, gives low-quality signal and cannot be used reliably with adult rats without drilling or thinning the skull.

We used simple and low-cost materials, such as natural rubber, plastic tubes and a metal stylet. A custom-made probe holder can be produced in few minutes and adapted to the experimental conditions. These probe holders can accommodate one or more deep-penetration LD probes, for classical single site monitoring on the ischemic core or for multiple-site monitoring in different arterial territories in the same hemisphere or across the two hemispheres. Many probe holders could be produced, chemically sterilized, and stored for future use. Veterinary-approved surgical glue (cyanoacrilate), accelerated by cold air, is used to attach the probe holder to the intact surface of the rat skull, according to the desired cranial coordinates. Finally, the probe set-up is further secured in place by common sutures.

The overall time of this LD probe set-up, after mastering this technique, is about $10 \mathrm{~min}$.

As shown in this video, we routinely monitor cerebral perfusion in the central MCA territory (LD probe 1: ischemic core) and in the peripheral MCA territory (LD probe 2: mainly a penumbral area). In our recent study we showed that the variability of blood flow changes in LD probe 2 (mean $52 \% \pm 16 \%$ SD, compared to the baseline) is higher compared to LD probe 1 (mean $31 \% \pm 6 \%$ SD, compared to the baseline) and may be used to predict stroke outcome ${ }^{4}$.

We may provide some trouble-shooting advices for researchers which would like to use our in-house developed system. At the beginning of the experiment, be careful to dry very well the skull surface (with Merbromin and cold air) before attaching the probe holder to prevent premature detachment. Moreover, be sure to apply the glue on the natural rubber, avoiding contact with the open end of the plastic tube and the optical surface of the LD probe, to prevent poor signal and potential damage to the probe. When tying the suture around the head of the animal, be careful in order to avoid airway obstruction (this is prevented by positioning the suture over the mandibular bone). After positioning and securing the probes, be careful not to traction probe cables when turning the animal in the supine position for cervical surgery; this step usually requires two people, one person holding the animal and a second person holding the probe cables and gently positioning them to the desired position. Finally, eventual blood contamination of the twin-fibre LD probe is easily managed following the cleaning instructions provided by the manufacturer.

Our optimized system for cerebral perfusion monitoring, as shown in this video, could provide an easier, more rapid and more reliable alternative to the probe set-up systems which are currently sold by commercial companies in this field. Moreover, we believe that the use of this system by other researchers may enhance the study of cerebral hemodynamics in the experimental stroke field, leading to the development of a new generation of cerebral collateral therapeutics.

\section{Disclosures}

The funding for the production of this video-article was provided by Moor Instruments Ltd (Axminster, Devon, UK), which produces the Laser Doppler instruments shown in our experimental study.

The experimental protocol was approved by the Committee on Animal Care of the University of Milano Bicocca, in accordance with the national guidelines on the use of laboratory animals (D.L. 116/1992) and the European Union Directive for animal experiments (2010/63/EU), under project license (n. 219/2011-B) from the Italian Ministry of Health. 


\section{Acknowledgements}

We thank Mrs. Caroline Robertson for the voice-over and Mrs. Elena Pirovano for her assistance in video production. This study was supported by the University of Milano Bicocca, "Fondo di Ateneo 2011".

\section{References}

1. Sutherland, B.A., Papadakis, M., Chen, R.L., \& Buchan, A.M. Cerebral blood flow alteration in neuroprotection following cerebral ischemia. J. Physiol. 589, 4105-4114 (2011).

2. Prinz, V. \& Endres, M. In: Modeling focal cerebral ischemia in rodents: Introduction and overview., Dirnagl, U., ed., Rodent models of stroke, Chapter 3, Human Press, (2010).

3. Schmid-Elsaesser, R., Zausinger, S., Hungerhuber, E., Baethmann, A., \& Reulen, H.J. A critical reevaluation of the intraluminal thread model of focal cerebral ischemia: evidence of inadvertent premature reperfusion and subarachnoid hemorrhage in rats by laser-Doppler flowmetry. Stroke. 29, 2162-2170 (1998).

4. Riva, M., Pappadà, G.B., et al. S. Hemodynamic monitoring of intracranial collateral flow predicts tissue and functional outcome in experimental ischemic stroke. Exp. Neurol. 233, 815-820 (2012).

5. Menon, B.K., Smith, E.E., et al. Regional leptomeningeal score on CT angiography predicts clinical and imaging outcomes in patients with acute anterior circulation occlusions. Am. J. Neuroradiol. 32, 1640-1645 (2011).

6. Bang, O.Y., Saver, et al. Collateral flow predicts response to endovascular therapy for acute ischemic stroke. Stroke. 42, 693-699 (2011).

7. Shuaib, A., Butcher, K., Mohammad, A.A., Saqqur, M., \& Liebeskind, D.S. Collateral blood vessels in acute ischaemic stroke: a potential therapeutic target. Lancet Neurol. 10, 909-921 (2011).

8. Liu, S., Zhen, G., Meloni, B.P., Campbell, K., \& Winn, H.R. Rodent Stroke Model Guidelines for preclinical stroke trials ( $1^{\text {st }}$ edition). J. Exp. Stroke Transl. Med. 2, 2-27 (2009).

9. Shepherd, A.P. \& Öberg, P.A. In: Laser-Doppler Blood Flowmetry., Kluwer Academic Publishers, (1990).

10. Uluç, K., Miranpuri, A., Kujoth, G.C., Aktüre, E., \& Başkaya, M.K. Focal Cerebral Ischemia Model by Endovascular Suture Occlusion of the Middle Cerebral Artery in the Rat. J. Vis. Exp. (48), e1978, doi:10.3791/1978 (2011).

11. Garcia, J.H., Wagner, S., Liu, K.F., \& Hu, X.J. Neurological deficit and extent of neuronal necrosis attributable to middle cerebral artery occlusion in rats. Statistical validation. Stroke. 26, 627-34 (1995). 